\title{
Analysis and recombination of multiple-charge-state beams from an electron cyclotron resonance ion source
}

\author{
P. N. Ostroumov, S. A. Kondrashev, B. Mustapha, and R. Scott \\ ANL, Argonne, Illinois 60439, USA \\ N. E. Vinogradov \\ Northern Illinois University, DeKalb, Illinois 60115, USA \\ (Received 14 September 2008; published 13 January 2009)
}

\begin{abstract}
To meet the beam power requirements for high-intensity ion linacs being proposed for rare isotope beam production and other nuclear physics applications, we have developed an injector system to extract, accelerate, analyze, and recombine multiple charge states of any heavy-ion beam. The injector consists of an electron cyclotron resonance ion source, a $100 \mathrm{kV}$ platform and an achromatic low-energy beam transport system. Two charge states of bismuth-209 $(20+$ and $21+)$ were successfully accelerated by the high-voltage platform potential, separated and perfectly recombined in the transverse phase space with $100 \%$ transmission. The perfect recombination and maximum transmission of the $\mathrm{cw}$ beam are essential for smooth injection into a subsequent $\mathrm{rf}$ accelerator.
\end{abstract}

DOI: 10.1103/PhysRevSTAB.12.010101

PACS numbers: 41.85.-p, 29.20.Ba, 29.25.Ni, 29.27.Eg

\section{INTRODUCTION}

Ion accelerators worldwide use only single charge state beams from the ion source. An electron cyclotron resonance ion source (ECRIS) is widely used as an injector of highly charged ions. Current state of the art ECRIS built using superconducting (SC) magnets has recently demonstrated $\sim 6 \mathrm{p} \mu \mathrm{A}$ of uranium ions with charge state $33+$ or $34+[1]$. Taking into account the acceleration and stripping efficiencies, the ion source intensity must be doubled to meet the power requirements for the proposed facility for rare isotope beams [2] and other nuclear physics applications based on high-intensity ion linacs. Even if the intensity of single charge state beams will be doubled in the future, the appropriate solution to simultaneously extract and accelerate multiple charge states of the desired heavyion relaxes the operational requirements of the ECR source. This solution is not appropriate for light ions due to larger charge states separation in the phase space (large $q / A$ separation, where $q$ is the ion charge state and $A$ is the mass number). Fortunately, ion sources produce enough single charge state intensity for light ions. In addition to future facilities, existing facilities [3] could benefit as well from the concept of multiple-charge-state acceleration.

In earlier studies [4-6], we proposed and demonstrated the concept of simultaneous acceleration of multiple charge states of a heavy-ion beam by a superconducting linac. Charge states within a $q / A$ range of $20 \%$ were accepted and accelerated. In this study we have designed and built a prototype multiple-charge-state injector system to demonstrate the possibility of extracting, analyzing, and combining several charge states of a heavy-ion beam from the ion source to the point of injection to an rf accelerator.
The injector consists of an ECRIS placed on a high-voltage (HV) platform and an achromatic low-energy beam transport (LEBT). The system was successfully tested for a two charge state bismuth beam. This technique can be applied for both the driver and postaccelerator in radioactive beam facilities. It is well recognized that the ECRIS and the electron beam ion source (EBIS) are effective charge breeders for radioactive beams [7]. The intensity of radioactive ions extracted either from an ECRIS or EBIS can be increased by combining several neighboring charge states into the same phase space area for further acceleration. This is especially important for rare isotope beams where doubling or tripling the intensity is critical for certain measurements.

\section{EXPERIMENTAL SETUP}

A 3D model of the injector is shown in Fig. 1. It consists of an ECR ion source, a $100-\mathrm{kV}$ platform, and an achromatic LEBT system based on two $60^{\circ}$ bending magnets. The stand-alone ECR ion source is built using all permanent magnets, it is described in more detail elsewhere [8]. The HV platform was designed and constructed to accelerate all ion species extracted from the ECR source to higher energy to suppress space-charge effects in the LEBT. The focusing is provided by electrostatic Einzel lenses and quadrupole triplets. Rotating wires are used for beam profile measurements. The beam emittance is measured by a specially developed scintillator-based pepperpot emittance probe described in the following section. The injector system allows us to accelerate all ion species up to $q \times 100 \mathrm{keV}$ total kinetic energy. 

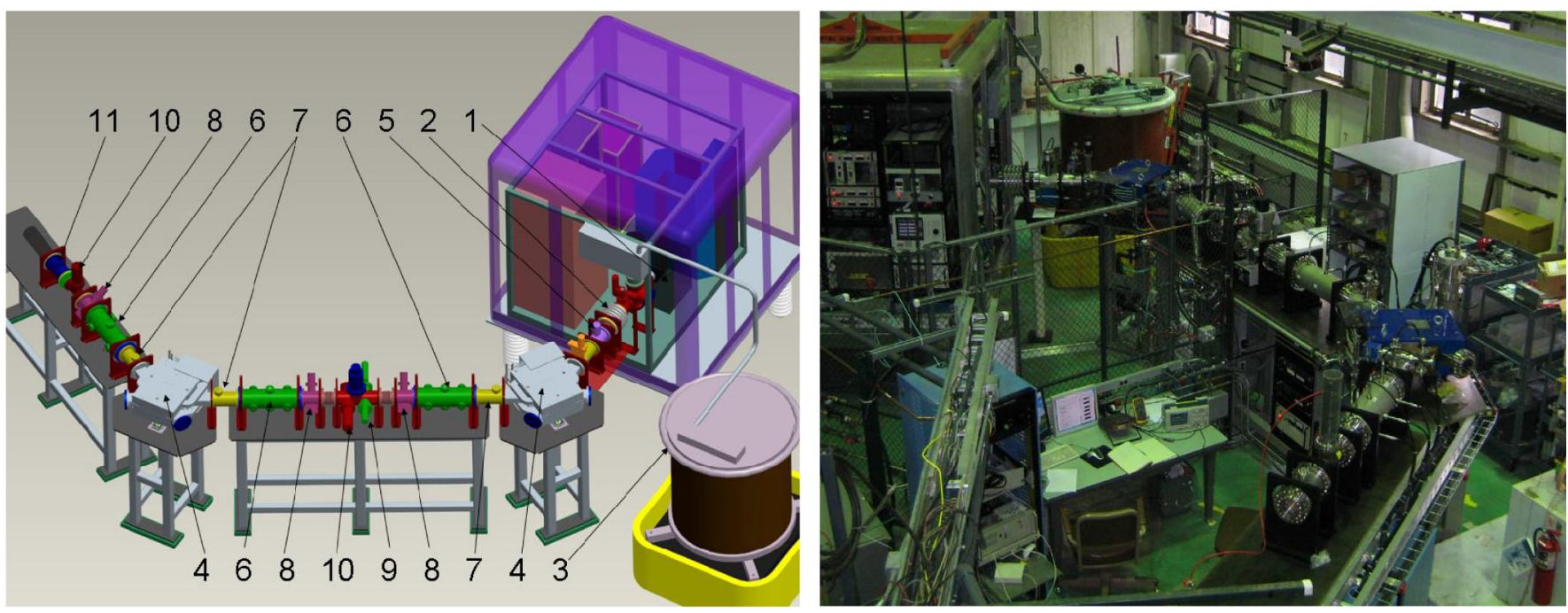

FIG. 1. (Color) General view of the injector. The picture on the left is the engineering model while the right picture is photo of the injector: 1, all permanent magnet ECRIS installed on $\mathrm{HV}$ platform; $2,75-\mathrm{kV}$ accelerating tube; 3 , isolation transformer; $4,60^{\circ}$ bending magnet; 5, Einzel lens; 6, electrostatic triplet; 7, electrostatic steering plates; 8, rotating wire scanner; 9, horizontal jaw slits; 10, Faraday cup; 11, emittance probe.

Our injector differs from any other ECR source on a HV platform currently used in various applications worldwide. Specifically, we extract all ion species available from the ECR source and analyze them after acceleration by the platform potential. For the purpose of these experiments, we use a bismuth ion beam which is relatively simple to produce using an oven heated to $550^{\circ} \mathrm{C}$. The ECR is equipped with two rf amplifiers set to 12.8 and $13.8 \mathrm{GHz}$ with total rf power up to $1.5 \mathrm{~kW}$. Oxygen is used as a support gas to produce higher charge states of ${ }^{209} \mathrm{Bi}$ ions.

The bismuth ion beam is first extracted by applying a $15 \mathrm{kV}$ source potential and then accelerated by a $75 \mathrm{kV}$ platform potential. To analyze the beam, a $36 \mathrm{~mm}$ aperture Faraday cup (FC) equipped with a suppression ring is installed downstream of the first $60^{\circ}$ magnet to record the beam current. After a few days of source conditioning and tuning, we were able to measure the distribution of ${ }^{209} \mathrm{Bi}$ ions. Figure 2 shows a typical spectrum for the different charge states extracted from the source. Depending on the operational parameters, a total current of $4 \mathrm{~mA}$ (source drain current) is extracted from the ECR. As can be seen from Fig. 2, the most intense beam is oxygen $2+$, which is used as a support gas in the source.

For the purpose of our experiment we are interested to work with ${ }^{209} \mathrm{Bi}^{20+}$ and ${ }^{209} \mathrm{Bi}^{21+}$ to reduce charge spread. When the ECR and bending magnet are tuned to transmit the bismuth ions, the unwanted bismuth charge states and light ion beams are intercepted by the vacuum chamber of the bending magnet and grounded apertures along the beam line. The required bismuth beam charge states and highest intensities are optimized by adjusting the ECRIS parameters such as support gas flow and biased disk potential. The $12.8 \mathrm{GHz}$ rf heater is set to the maximum
$950 \mathrm{~W}$ power and the second $13.8 \mathrm{GHz}$ rf source is set to generate $300-400 \mathrm{~W}$ power which is optimal to obtain ion beams with stable parameters. By adjusting the abovementioned parameters, for a short period of time (several hours), the ECR source can be tuned to produce up to $\sim 2.0 \mathrm{p} \mu \mathrm{A}$ of bismuth in each charge state $20+$ and $21+$. However, this mode of operation is unstable and cannot be reproduced in day-to-day operation. Our long-term operation experience showed that the most stable operation of the ECR being used and the lowest beam emittances can be achieved for beam intensities of $\sim 1.0 \mathrm{p} \mu \mathrm{A}$ for the charge states $20+$ and $21+$ of bismuth.

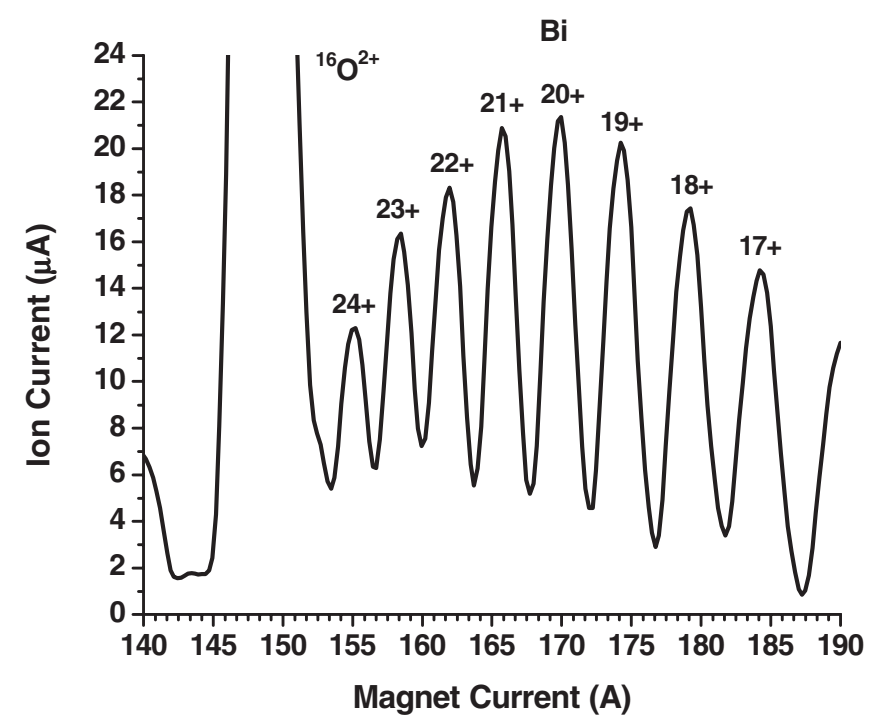

FIG. 2. Bismuth beam intensities for different charge states measured at $90 \mathrm{kV}$. 


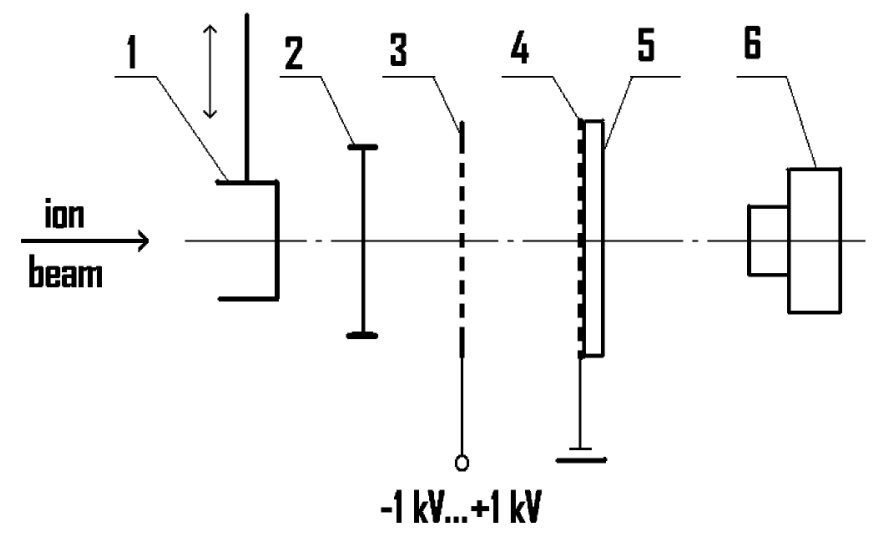

FIG. 3. Simplified scheme of the PPSS emittance probe: 1, movable FC; 2, fast iris-type shutter; 3, pepper-pot plate; 4, mesh; 5, CsI (Tl) crystal; 6, CCD camera.

\section{EMITTANCE PROBE}

The pepper-pot-scintillator screen (PPSS) method can be effectively used to measure emittances of cw beams generated by ECR ion sources [9]. The PPSS assembly, shown in Fig. 3, is located at the end of the LEBT downstream of the FC. The pepper-pot plate is $380 \mu \mathrm{m}$ thick and has 415 pinholes with $100 \mu \mathrm{m}$ diameter and 3-mm spacing between holes horizontally and vertically covering a working area with $70 \mathrm{~mm}$ diameter. The CsI (Tl) crystal was chosen as a scintillator screen because it has shown the highest sensitivity and long lifetime for accelerated beams. The diameter and thickness of the crystal are 80 and $3 \mathrm{~mm}$, respectively. A grounded fine metal mesh with transparency above $90 \%$ is attached to the crystal surface irradiated by ions to prevent potential rise caused by ion beam charge.

It was found that $\mathrm{CsI}(\mathrm{Tl})$ has a high sensitivity for a variety of ion species from protons to heavy ions. Pepperpot images were at a recordable level for proton and bismuth beams with current densities below $1 \mu \mathrm{A} / \mathrm{cm}^{2}$.

The distance between the pepper-pot and scintillator screen is $100 \mathrm{~mm}$. An IMI TECH IMB-147FT monochrome digital CCD camera connected to a PC was used to acquire and save beam images. The pepper-pot plate is isolated from ground and its potential can be varied in the range of $\pm 1 \mathrm{kV}$ to study the effect of secondary electrons on the measured emittance. The FC was used as a slow shutter and detector of the ion beam current.

\section{INJECTOR DESIGN AND SIMULATION}

The ECR ion source generates multicomponent ion beams with total intensity up to $4 \mathrm{~mA}$. The main purpose of the LEBT is to select a particular ion specie and match it into the following rf accelerator. The selection of the required ion specie is usually performed by adjustable horizontal jaw slits located in the high-dispersion area of the LEBT downstream of the first bending magnet. Several charge states of ion beam downstream of the selection slits can be recombined into a beam with the same transverse phase space if the LEBT is an achromatic system. Indeed, the achromatic bending system maintains the beam transverse emittance unchanged for all particles with momentum $p$ in the range of $p_{0}\left(1-\frac{\Delta p_{-}}{p_{0}}\right) \leq p \leq p_{0}\left(1+\frac{\Delta p_{+}}{p_{0}}\right)$, where $p_{0}$ is the central momentum, $\Delta p_{-} / p_{0}$ and $\Delta p_{+} / p_{0}$ are the minimum and maximum relative momentum deviation in the beam, respectively. If the bending system is achromatic, it will provide similar transverse emittance transformation for ions with charge states between $q_{0}+\Delta q$ and $q_{0}-\Delta q$ and equivalent momentum spread

$$
\frac{\Delta p_{-}}{p_{0}}=\frac{\Delta q}{q_{0}+\Delta q}, \quad \frac{\Delta p_{+}}{p_{0}}=\frac{\Delta q}{q_{0}-\Delta q} .
$$

The standard beam optics computer code COSY [10] was used to design and optimize the original layout of the LEBT by taking into account terms through third order. However, COSY does not include space-charge effects. Therefore, numerical studies of beam dynamics in the LEBT have been performed using the multiparticle code TRACK [11]. TRACK can simulate a multiple-component ion beam through precalculated external 3D fields taking into account self-consistent space-charge effects. The magnetic field of the ECR extraction region and electrostatic fields in the initial part of the beam line that consists of a puller electrode, Einzel lens, and grounded electrodes were calculated using the CST-STUDIO software [12]. 3D representation of magnetic field including fringe fields was implemented using genuine configuration of the $60^{\circ}$ bending magnet. Similarly, the electric fields of the triplets were implemented into TRACK. TRACK simulations have shown that the bending magnet must provide strong vertical focusing in order to compensate for space-charge forces. For the space-charge calculation, we assume 50\% charge compensation along the LEBT except in electrostatic elements where we use 0\%. The LEBT system shown in Fig. 1 is achromatic providing $\sim 25 \mathrm{~mm}$ separation on the slits for neighboring charge states of bismuth beam tuned for $20+$ as the central charge state.

The exact phase space distribution of ion beams extracted from the ECR source is very complicated (see for example [13,14]) and can not be reproduced with any available computer code. For the initial design of the LEBT we have used simplified, axial-symmetric multicomponent ion beams with the parameters best fitted to the measurements performed downstream of a $90^{\circ}$ magnet [15]. For the tuning and operation of the actual LEBT system, we have developed and applied specific optimization algorithms within the code TRACK. These algorithms use the measured data such as the horizontal $(\mathrm{H})$ and vertical $(\mathrm{V})$ beam profiles from the wire scanners and $\mathrm{H}$ profiles from the slits to adapt the computer model to the actual beam line. The first optimization step is to determine 
the beam emittance and Twiss parameters at the source that reproduces the measured beam profiles after the first bending magnet. The second step is to optimize the setting of nine quadrupoles to provide (a) no angular dispersion on the slits and (b) 100\% beam transmission downstream of the slits.

\section{EXPERIMENTAL RESULTS}

Figure 4 shows the measured (curves) $\mathrm{H}$ and $\mathrm{V}$ profiles of a two charge state bismuth beam $(20+, 21+)$ on the wire scanner behind the first magnet. We can see clearly that the TRACK fit (histograms) reproduced reasonably well the measured profiles. This fit allowed the extraction of the initial beam emittance and Twiss parameters at the source to use for further tuning and operation of the LEBT. For realistic beam dynamics simulations we track 17 beams simultaneously ( $\mathrm{O}$ and $\mathrm{Bi}$ as in Fig. 2). The quadrupole settings were then optimized using TRACK to recombine the ${ }^{209} \mathrm{Bi}^{20+}$ and ${ }^{209} \mathrm{Bi}^{21+}$ beams with $q_{0}=20.5+$ as the central charge state. Setting the values obtained by TRACK
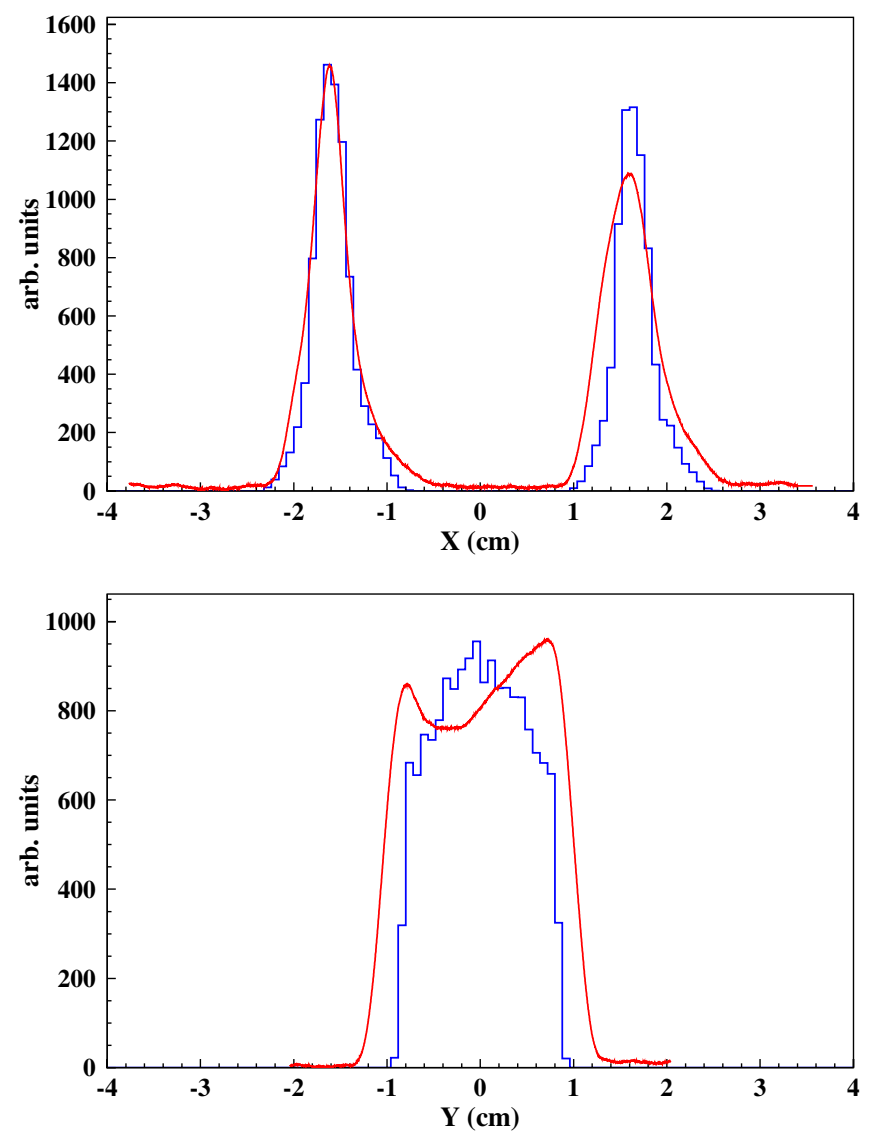

FIG. 4. (Color) The curves are the horizontal (top) and vertical (bottom) beam profiles for a two charge state bismuth beam $(20+, 21+)$ measured at the wire scanner behind the first magnet. The histograms are the results of a TRACK fit to extract the initial beam conditions at the source.

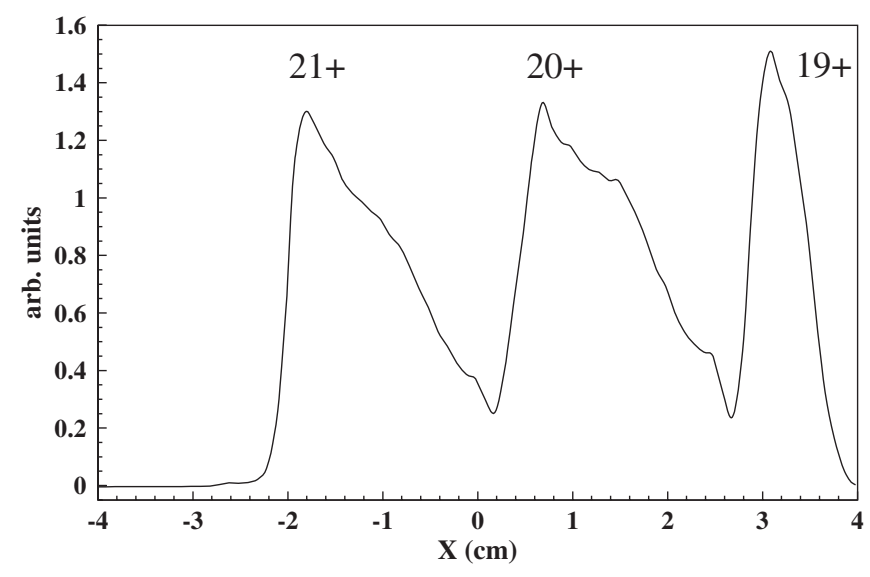

FIG. 5. Measured bismuth beam profiles at the slits.

we noticed a very good agreement between the simulation and the actual measurements.

After we obtained initial beam parameters upstream of the first magnet, the LEBT was retuned to zero angular momentum dispersion at the separation slits placed at the midpoint of the LEBT. Figure 5 shows the horizontal beam profile at the slits. We notice a good separation of the charge states which is important to cleanly select a single or a multiple-charge-state beam. At the end of the LEBT, the beam emittance was measured for the following three different operation modes: (1) only ${ }^{209} \mathrm{Bi}^{20+}$ is selected by the slits; (2) only ${ }^{209} \mathrm{Bi}^{21+}$ is selected; (3) both ${ }^{209} \mathrm{Bi}^{20+}$ and ${ }^{209} \mathrm{Bi}^{21+}$ are selected. The beam parameters are given in Table I. The focusing triplet upstream of the emittance probe is tuned to provide $\sim 30 \mathrm{~mm}$ beam diameter on the emittance probe. Only several percent adjustment of quadrupole setting with respect to the precalculated values is required in order to combine the dual charge state bismuth beam. $100 \%$ beam transmission from FC-1 to FC-2 has been achieved with typical beam currents of

$$
\begin{gathered}
I_{20+}=(20.9 \pm 0.2) \mu \mathrm{A}, \quad I_{21+}=(21.3 \pm 0.2) \mu \mathrm{A} \\
I_{20+, 21+}=(42.1 \pm 0.4) \mu \mathrm{A} .
\end{gathered}
$$

Figure 6 shows the H- and V-beam profiles on the wire scanner downstream of the second magnet while the pepper-pot images of single- and dual-charge-state bis-

TABLE I. The rms normalized emittances and Twiss parameters of individual charge states $(20+, 21+)$ and combined bismuth beams at the end of the LEBT.

\begin{tabular}{lccc}
\hline \hline Parameter & ${ }^{209} \mathrm{Bi}^{20+}$ & ${ }^{209} \mathrm{Bi}^{21+}$ & ${ }^{209} \mathrm{Bi}^{20+}+{ }^{209} \mathrm{Bi}^{21+}$ \\
\hline$\varepsilon_{\mathrm{X}}(\pi \mu \mathrm{m})$ & 0.092 & 0.081 & 0.087 \\
$\alpha_{\mathrm{X}}$ & 0.816 & -0.125 & 0.259 \\
$\beta_{\mathrm{X}}(\mathrm{mm} / \mathrm{mrad})$ & 2.93 & 3.17 & 2.68 \\
$\varepsilon_{\mathrm{Y}}(\pi \mu \mathrm{m})$ & 0.055 & 0.059 & 0.057 \\
$\alpha_{\mathrm{Y}}$ & -2.92 & -3.33 & -3.32 \\
$\beta_{\mathrm{Y}}(\mathrm{mm} / \mathrm{mrad})$ & 0.779 & 0.902 & 0.895 \\
\hline \hline
\end{tabular}



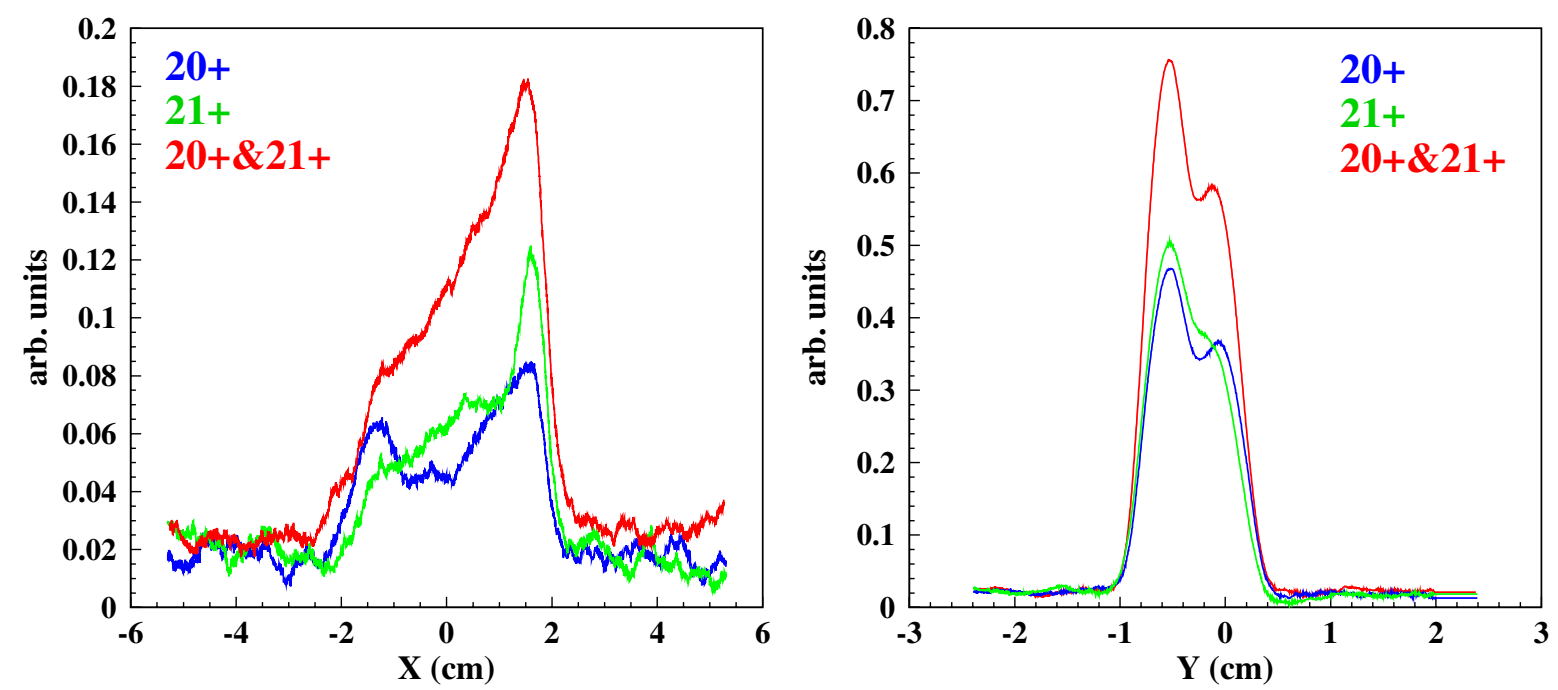

FIG. 6. (Color) Measured bismuth beam profiles on the wire scanner downstream of the second bending magnet.

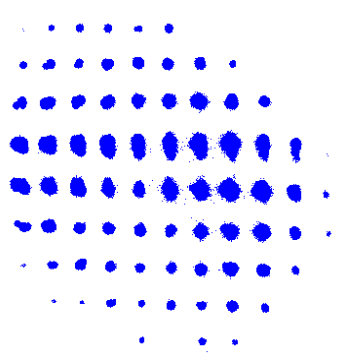

FIG. 7. (Color) Pepper-pot image of the combined bismuth beam with charge states $20+$ and $21+$ (on the left) and superimposed images of ${ }^{209} \mathrm{Bi}^{20+}$ (blue) and ${ }^{209} \mathrm{Bi}^{21+}$ (red) beams transported individually (on the right).

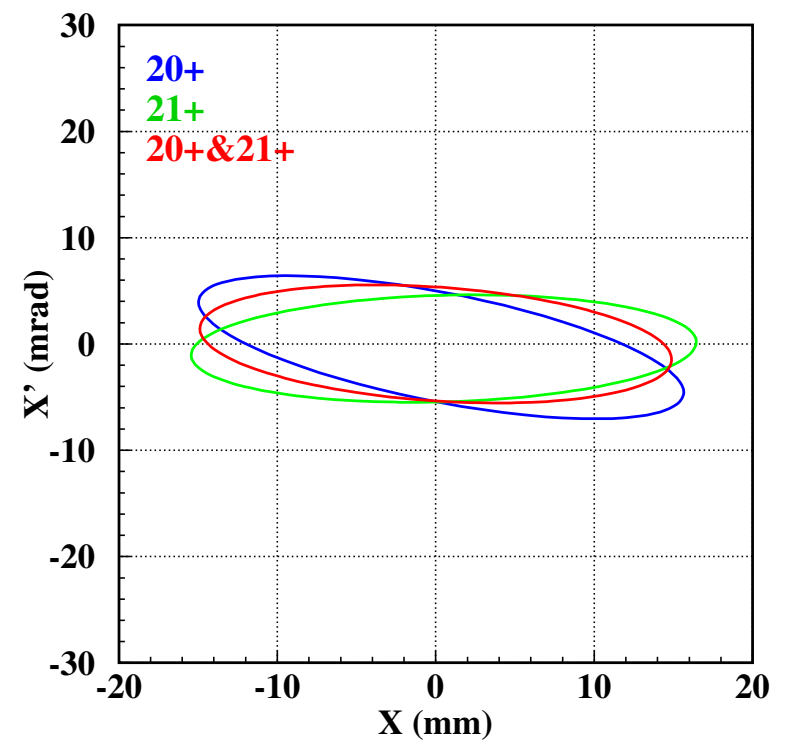

muth beam are shown in Fig. 7. The phase space ellipses extracted from the pepper-pot images for the individual charge states and combined bismuth beams are shown in Fig. 8. As can be seen from these figures, two charge states of bismuth beam are perfectly combined into the same phase space area. The emittances and Twiss parameters of the beam derived from the pepper-pot data for the three operation modes of the LEBT are listed in Table I. The rms emittance of dual charge state beam is close to the emittance of the individual charge state beams while the mismatch factor [16] of each charge state beam with respect to the dual charge state beam is less than $30 \%$. This latter can be further reduced by applying a sextupole correction as suggested by higher order beam optics calculations. As is

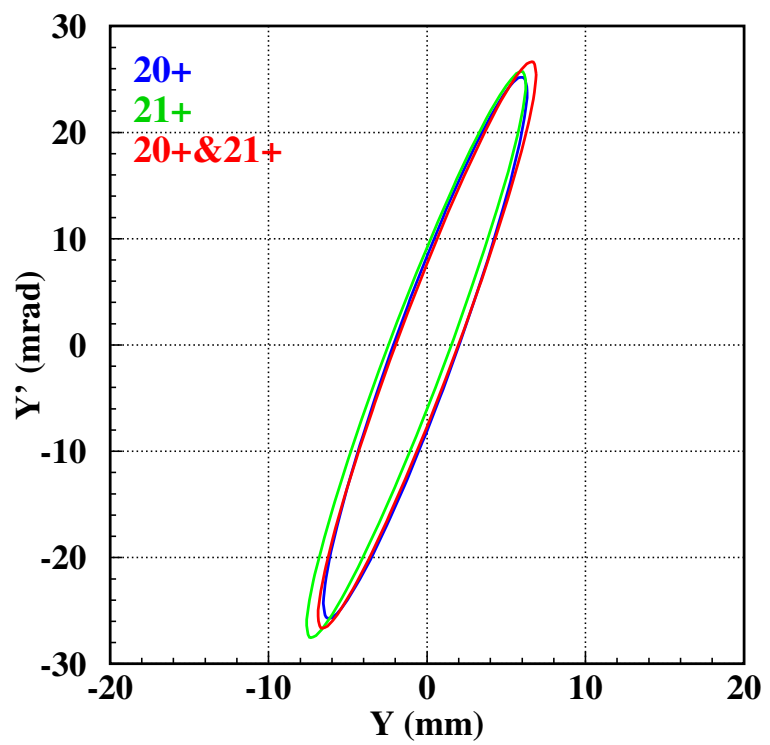

FIG. 8. (Color) Beam phase space ellipses $(4 \times$ rms emittances $)$ extracted from the pepper-pot images for individual charge states and combined bismuth beams. 
seen from Table I, the rms emittance of dual charge state beam is smaller than the rms emittance of the individual beam. This can be explained by the fact that emittance measurements have been done with automatic adjustment of the CCD camera gain [17]. Because of limited dynamic range of the emittance meter, some information of the particle distribution below the threshold can be lost.

\section{CONCLUSION}

Two charge states of bismuth ions from an ECR ion source have been extracted, accelerated to an energy of $1.8 \mathrm{MeV}$, separated and then recombined into a high quality beam ready for further acceleration. This technique allows us to double the intensity of heavy-ion beams in high-intensity linacs for future radioactive beam facilities and other nuclear physics applications. Another important application of the concept of multiple-charge-state extraction and acceleration is in postaccelerators of radioactive ions based on charge breeders. The intensity of rare isotope beams can be doubled or tripled which could be critical for certain measurements.

\section{ACKNOWLEDGMENTS}

This work was supported by the U.S. Department of Energy, Office of Nuclear Physics, under Contract No. DEAC02-06CH11357.
[1] D. Leitner et al., Rev. Sci. Instrum. 79, 02 C710 (2008).

[2] J. A. Nolen, Nucl. Phys. A787, 84c (2007).

[3] H. Sakurai, Nucl. Phys. A805, 526c (2008).

[4] P. N. Ostroumov et al., in Proceedings of the 20th International Linac Conference, Monterey, CA, 2000, edited by A. W. Chao, p. 202 (SLAC-R-561).

[5] P. N. Ostroumov et al., Phys. Rev. Lett. 86, 2798 (2001).

[6] A. A. Kolomiets et al., in Proceedings of the 2003 Particle Accelerator Conference, Portland, OR, 2003, edited by J. Chew (IEEE, New York, 2003), p. 2875.

[7] T. Lamy, J. Angot, and T. Thuillier, Rev. Sci. Instrum. 79, 02A909 (2008).

[8] D.Z. Xie, Rev. Sci. Instrum. 73, 531 (2002).

[9] S. A. Kondrashev et al., in Proceedings of the 2007 Particle Accelerator Conference, Albuquerque, New Mexico, 2007, edited by S. Schriber (IEEE, Albuquerque, New Mexico, 2007), p. 1658.

[10] http://bt.pa.msu.edu/index_files/cosy.htm.

[11] http://www.phy.anl.gov/atlas/TRACK.

[12] http://www.cst.com/.

[13] P. Spadtke et al., Rev. Sci. Instrum. 79, 02 B716 (2008).

[14] D. S. Todd et al., Rev. Sci. Instrum. 79, 02 A316 (2008).

[15] N. E. Vinogradov et al., in Proceedings of the 2006 Linear Accelerator Conference, Knoxville TN, 2006, p. 336.

[16] T. Wangler, RF Linear Accelerators, Wiley Series in Beam Physics and Accelerator Technology (Wiley-VCH Verlag GmbH \& Co. KGaA, Weinheim, 2004), p. 217.

[17] S. A. Kondrashev et al., paper TUP085 in Proceedings of the 2008 Linear Accelerator Conference, Victoria BC, Canada, 2008. 